to force the polymer to swell in organic solvents, he and Inganäs investigated the behavior of PEDOT-PSS blends with PEO, which are known to form conductive PEDOT-PSS networks in a PEO matrix that could provide space for ionic movements.

Films of the polymer blends were cast onto gold-coated silicon wafers from aqueous dispersions and used as active electrodes. Cyclic voltammetry indicated that the PEDOT-PSS/PEO blends show the expected higher ionic mobility. Although the measured current is lower than for swollen hydrogels, the two values become comparable if they are normalized to the mass of PEDOT-PSS that is present in each electrode. This indicates similar efficiencies for both systems. The efficiency of the blend electrode increases with decreasing PEDOT-PSS content (down to about 25\%), showing that the high compatibility of the two polymers allows the formation of a network morphology even at low PEDOT-PSS concentrations. Supercapacitor measurements gave high energy densities for the blend electrodes even at power densities five times higher than the drop-off for pure PEDOT-PSS electrodes. "These power densities were calculated using the total weight of the blend", Ghosh said. "This means that we get higher energies with a smaller amount of redox material."

"The increase in ionic mobility in the PEDOT-PSS blend with inclusion of PEO can be ascribed to the intrinsic ionic conductivity of the latter polymer as well as to the swelling of the PEO phase in the acetonitrile solution, creating space for ionic movement," Inganäs said. The researchers reported that the comparative experiments with blended and unblended PEDOT-PSS electrodes in a solid-state cell suggest that "the intrinsic ionic conductivity of PEO does contribute to the enhancement of ionic mobility."

Currently, the group is working "on better crosslinking of the electronically conducting PEDOT-PSS phase and on loading the material with more energyrich material to increase the energy density, keeping the network morphology and hence the ionic mobility intact," said Ghosh. In the hydrogels, this has been achieved by introduction of polypyrrole, a second conducting polymer.

CORA LIND

\section{Versatile Pattern Transfer Process for Polymers Developed}

Researchers from the University of Massachusetts and the University of Groningen, The Netherlands, have reported a nanoscopic pattern transfer phenomenon. Erik Schaffer, a graduate student at Groningen, and Thomas Thurn-Albrecht, a postdoctoral researcher at Massachusetts, placed a thin film of polystyrene atop an electrode. They placed a second electrode above the film, leaving an air gap between the film and the top electrode. They then heated the polystyrene, liquefying it, and placed a small voltage on the electrodes.

With time, as reported in the February 24 issue of Nature, the surface of the film appeared pockmarked. Tom Russell, in polymer science and engineering at Massachusetts, said that the electric field amplified waves on the liquid's surface. The waves were increasingly amplified and eventually were pulled to the top electrode. The phenomenon appears under the microscope as a dark ring on a light background. As time passed, more and more circles appeared. The circles were all the same size, and appeared at a precise distance from one another.

Russell said that the phenomenon occurs because of the interaction of four competing forces. Those forces include the electrical force, which pulls the liquid toward the top electrode; the surface energy of the liquid, which wants the liquid to lie flat; the viscosity of the liquid as crests and valleys form and the liquid moves; and the effects of atmospheric pressure. "It doesn't happen helter-skelter," he said. "It happened at very distinct distances that represents a delicate balance between all of these forces."

Through a process called pattern transfer, the researchers can "imprint" a film with a very specific design. In this process, an electrode is etched with a master pattern. The master electrode has a topography of "hills" and "valleys." When a voltage is applied, the film responds most strongly to the closest portions of the electrode, creating a replica of the master design on the polymer film. The researchers said that their findings have implications in paving the way for stillsmaller integrated circuits, magnetic storage in computers, and on-chip sensorsall of this without the use of chemicals.

\section{Additional $\mathrm{CeO}_{2}$ Buffer Layer Improves Performance of Superconducting Tape}

The microstructural development of a superconducting tape (coated conductor) consisting of a layer of $\mathrm{YBa}_{2} \mathrm{Cu}_{3} \mathrm{O}_{\mathrm{y}}(\mathrm{Y}-123)$ superconducting material (1-3- $\mu \mathrm{m}$ thick) on an Inconel 625 substrate $100-\mu m$ thick with a layer of yittria-stabilized zirconia (YSZ) $0.8-\mu \mathrm{m}$ thick and $\mathrm{CeO}_{2}$ buffer lay- ers ( $\sim 0.03-\mu$ m thick) was studied by researchers at the Los Alamos National Laboratory (LANL). They reported in the May 2000 issue of the Journal of Materials Research that an additional buffer layer is useful "for removing the misfit strain from the interface with the superconductor and limiting the extent of interfacial reactions with Y-123."

Since its discovery, Y-123 has been actively investigated for high critical current and high current density applications at liquid nitrogen temperatures. The epitaxially prepared materials studied at LANL exhibit the best critical current and current density in a $1 \mathrm{~m} \mathrm{Y-123}$ tape produced to date. These favorable characteristics are believed to result from the use of two buffer layers between the substrate and the superconducting film. The YSZ buffer layer improves performance by preventing the $\mathrm{Ni}$ and $\mathrm{Cr}$ from the substrate from contaminating the Y-123 layer and providing a biaxially aligned template for its growth. The $\mathrm{CeO}_{2}$ buffer layer is believed to improve conductor performance by removing the lattice mismatch strain between the YSZ and Y-123 layers. To extend the current technology to the production of conductors longer than $1 \mathrm{~m}$, and possibly improve conductor performance, the microstructural properties of the conductor during processing need to be investigated. This study detected and explained a number of such features.

A $\mathrm{Cr}_{2} \mathrm{O}_{3}$ layer was detected between the substrate and the YSZ layers by electron diffraction and energy dispersive spectroscopy. The researchers speculate that this layer formed in situ during the high temperature deposition of the $\mathrm{CeO}_{2}$ and Y-123 layers and not during the room temperature deposition of the YSZ. The researchers believe that it is the $\mathrm{Cr}_{2} \mathrm{O}_{3}$ layer that prevents $\mathrm{Ni}$ and $\mathrm{Cr}$ from the Inconel from contaminating the Y-123, as neither metal could be detected above the $\mathrm{Cr}_{2} \mathrm{O}_{3}$. This is an important finding because it proves that the Inconel substrate is compatible with the YSZ layer. This is advantageous because YSZ is the only material known that can be reliably deposited as a biaxially textured film for Y-123 deposition.

No evidence for a reaction between YSZ and $\mathrm{CeO}_{2}$ was detected; however, there was evidence of misfit dislocation between the layers. According to the researchers, since Y-123 is closely lattice matched to $\mathrm{CeO}_{2}$, the extra buffer layer apparently moves the misfit strain away from the Y-123 film. The researchers reported, "A volume constraint imposed by the overlying Y-123 film may act as a kinetic barrier and limit the extent of the 